\title{
Associations between bovine milk protein genotypes and haplotypes and the breeding value of Polish Black-and-White bulls*
}

\author{
S. Kamiński ${ }^{1}$, J. Rymkiewicz-Schymezyk ${ }^{2}$, E. Wójcik' and A. Ruść'
}

\author{
University of Warmia and Mazury in Olsztyn, \\ 'Department of Animal Genetics, \\ ${ }^{2}$ Department of Breeding Methods and Animal Improvement \\ Oczapowskiego 5, 10-957 Olsztyn, Poland
}

(Received 18 January 2002; accepted 9 May 2002)

\begin{abstract}
One hundred forty three proven Polish Black-and-White bulls were genotyped for 6 polymorphic sites located within 4 milk protein genes (LGB-R promoter, LGB exon IV, CASK-R promoter, CASK exon IV, LALBA 5' flanking region and CSNIS1 promoter). All bulls were divided into groups, including animals with the same genotypes, intragenic haplotypes and combinations of genotypes. For each group, the arithmetic mean and standard deviation for Type Production Index (TPI) and Predicted Transmitting Ability (PTA) (milk kg, protein $\mathrm{kg}$, protein $\%$, fat $\mathrm{kg}$, fat $\%$ ) were calculated. These data were then used for analysis of variance to find possible associations between identificd polymorphic sites and TPI and PTA values. Significant differences $(\mathrm{P}<0.01)$ were found within different genotypes of CASK, LGB, LALBA, intragenic haplotypes CASK/CASK-R, combinations of genotypes CASK/LALBA, CASK/LGB, LGB/LALBA, CASK/LGB/LALBA and TPI. Within these groups, the highest TPI value was found for bulls carrying the following genotypes: CASK/ LGB/LALBA AA/AA/PM, CASK/LGB AA/AA, CASK/CASK-R AA/MM, LGB/LALBA AA/ MM, LGB AA, CASK/LALBA AA/MM and LALBA MM. Also, associations between genotypes and combinations of genotypes and PTA for protein $\mathrm{kg}$, fat $\mathrm{kg}$, fat \%, protein $\%$ and milk $\mathrm{kg}$ were found.
\end{abstract}

KEY WORDS: bulls, milk protein, genotypes, haplotypes, PTI, TPI

- Supported by the State Committee for Scientific Rescarch, Grant No. 6 P06D 00620 and PBZ-KBN-036/P06/2000/05 


\section{INTRODUCTION}

Bovine milk protein genes are located on three chromosomes: the cluster of four casein genes (alpha S1-CSN1S1, alpha S2 - CSN1S2, beta - CSN2 and kappaCSN3) on chromosome 6 (Grosclaude et al., 1972; Ferretti et al., 1990; Threadgill and Womack, 1990; Lien et al., 1993; Rijnkels et al., 1997), the beta-lactoglobulin (LGB) on chromosome 11 (Hayes and Petit, 1993; Eggen and Fries, 1995) and alpha-lactalbumin (LALBA) on chromosome 5 (Hayes et al., 1993). Studies on the significance of milk protein polymorphisms have been carried out by many authors. Most of these studies were conducted on a single milk protein loci and generally found positive effects of kappa-casein allele B and beta-lactoglobulin allele B on different milk performance traits and technological properties of milk (Mao et al., 1992; Bovenhuis and Weller, 1994; Walawski et al., 1994; Ng-Kwai-Hang, 1997). Another group of investigations used the casein haplotypes as a genetic marker of milk performance traits, e. g., in Norwegian cattle (Lien et al. 1995), in Ayrshire (Velmala et al., 1995, 1999; Ikonen et al., 2001) and Swiss Brown (Braunschweig et al., 2000). These studies confirmed the occurrence of QTL for some milk performance traits (mostly protein \%) on chromosome 6 and the utility of milk protein genes (mostly casein haplotypes) as genetic markers of these traits for different breeds.

In this paper, six polymorphisms identified in 4 milk protein loci were combined and analysed for associations between individual genotypes, combinations of genotypes and haplotypes and breeding value of Polish Black-and-White bulls.

\section{MATERIAL AND METHODS}

One hundred forty three proven Polish Black-and-White bulls were included in the analysis. One portion of semen was used to isolate genomic DNA by the MasterPure DNA Purification Kit (Epicentre). DNA was used to amplify 6 PCR products, namely: a 208 bp fragment of LGB promoter, designated LGB-R (Kamiński and Zabolewicz, 1998), a 240 bp fragment of LGB exon IV, designated LGB (Kamiński and Zabolewicz, 1997), a 214 bp fragment of CASK promoter, designated CASK-R (Kamiński, 1996), a 273 bp fragment of CASK exon IV-intron IV, designated CASK (Kamiński, 1998), a 483 bp fragment of LALBA 5' flanking region, designated LALBA (Kamiński, 1999) and a 218 bp fragment of CSN1S1 promoter, designated CSN1S1 (Kamiński and Józefowicz, 2000). The breeding values of bulls were collected from the official ranking list of proven bulls published bi-annually by the National Animal Breeding Centre in Warsaw (Poland). These data included the Predicted Transmitting Ability (PTA) values for: milk yield (kg), fat content (\%), fat yield $(\mathrm{kg})$, protein yield $(\mathrm{kg})$, protein content $(\%)$, and Type Production Index. All 
bulls were divided into groups including animals with the same genotypes, intragenic haplotypes and combinations of genotypes. For each group, the arithmetic mean and standard deviation for TPI and PTA values were calculated. These data were then used to test the significance of differences between the means for TPI or PTA value for the following factors:

1. single genotypes: LGB (AA, AB, BB), LGB-R (R1, R2, R3, R9), CASK-R (PP, PM, MM), CASK (AA, AB), LALBA (PP, PM, MM) and CSN1S1 (R2, R3), 2. intragenic haplotypes: LGB/LGB-R (AA/R3, AB/R2, BB/R2, BB/R1), CASK/ CASK-R (AA/MM, AA/PM, AA/PP, AB/PM, AB/PP),

3. combinations of genotypes, e.g., CASK/LALBA, CASK/LGB, LGB/LALBA, CASK/LGB/LALBA, etc.

Statistical analysis was conducted by using the F-test and Duncan test incorporated in the computer program STATISTICA for Windows (STATSOFT 1995). Single genotypes, intragenic haplotypes and combinations of genotypes which were identified in less than 5 bulls were cxcluded from the statistical analysis. Because of the number of bulls, some theoretically possible intragenic haplotypes and combinations of genotypes were not identified within the population under investigation.

\section{RESULTS}

Statistical analysis revealed significant associations $(\mathrm{P}<0.01)$ between TPI, PTA values and:

1. single genotypes CASK, LGB and LALBA (Table 1),

2. intragenic haplotype CASK/CASK-R (Table 2),

3. combinations of genotypes CASK/LALBA (Table 3), CASK/LGB (Table 4), LGB/LALBA (Table 5), CASK/LGB/LALBA (Table 6).

Most associations were found for Type Prediction Index (calculated for Polish Black-and-White as TPI $=\mathrm{kg}$ fat $+2 \times \mathrm{kg}$ protein $)$. Significant differences $(\mathrm{P}<0.01)$ were found within different genotypes of CASK, LGB, LALBA, intragenic haplotypes CASK/CASK-R, combinations of genotypes CASK/LALBA, CASK/LGB, LGB/LALBA and CASK/LGB/LALBA. Within these groups the highest TPI was found for bulls carrying the following genotypes: CASK/LGB/LALBA AA/ AA/PM (58.4), CASK/LGB AA/AA (56.8), CASK/CASK-R AA/MM (52.7), LGB/LALBA AA/MM (51.5), LGB AA (48.1), CASK/LALBA AA/MM (42.1) and LALBA MM (40.4) (Figure 1).

PTA for protein $\mathrm{kg}$ was found to be associated with following genotypes CASK/ LGB/LALBA, CASK/LGB, LGB/LALBA and LGB, showing the highest values for AA/AA/PM (16.5), AA/AA (16.4), AA/MM (15.2) and AA (14.1), respectively (Figure 2). 
TABLE 1

Means and standard deviations of PTI and PTA values of animals with different CASK, LGB and LALBA genotypes

\begin{tabular}{|c|c|c|c|c|c|c|c|}
\hline \multirow{2}{*}{\multicolumn{2}{|c|}{ Genotype }} & \multirow[b]{2}{*}{ TPI } & \multicolumn{5}{|c|}{ Predicted Transmitting Ability } \\
\hline & & & $\begin{array}{c}\text { Milk } \\
\text { kg }\end{array}$ & $\begin{array}{l}\text { Fat } \\
\mathrm{kg}\end{array}$ & $\begin{array}{c}\text { Fat } \\
\%\end{array}$ & $\begin{array}{c}\text { Protein } \\
\mathrm{kg}\end{array}$ & $\begin{array}{c}\text { Protein } \\
\%\end{array}$ \\
\hline \multicolumn{8}{|l|}{ CASK } \\
\hline $\mathrm{AA}$ & $\mathrm{n}=94$ & $\begin{array}{r}37.80 \\
\pm 25.45\end{array}$ & $\begin{array}{r}352.70 \\
\pm 286.11\end{array}$ & $\begin{array}{r}17.10 \\
\pm 11.15\end{array}$ & $\begin{array}{r}0.470 \\
\pm 4.022\end{array}$ & $\begin{array}{r}10.40 \\
\pm 7.89\end{array}$ & $\begin{array}{l}-0.010^{\wedge} \\
\pm 0.084\end{array}$ \\
\hline $\mathrm{AB}$ & $n=47$ & $\begin{array}{r}32.30 \\
\pm 23.14\end{array}$ & $\begin{array}{r}238.90 \\
\pm 272.13\end{array}$ & $\begin{array}{r}14.60 \\
\pm 10.82\end{array}$ & $\begin{array}{r}0.100 \\
\pm 0.210\end{array}$ & $\begin{array}{r}8.90 \\
\pm 6.93\end{array}$ & $\begin{aligned} & 0.030^{\mathrm{B}} \\
\pm & 0.094\end{aligned}$ \\
\hline \multicolumn{8}{|l|}{ LGB } \\
\hline $\mathrm{AA}$ & $n=25$ & $\begin{aligned} & 48.10^{\wedge} \\
\pm & 23.72\end{aligned}$ & $\begin{array}{r}427.30 \\
\pm 257.34\end{array}$ & $\begin{array}{r}19.80 \\
\pm 11.15\end{array}$ & $\begin{array}{r}0.040 \\
\pm 0.204\end{array}$ & $\begin{aligned} & 14.10^{\wedge} \\
\pm & 7.02\end{aligned}$ & $\begin{array}{r}0.020 \\
\pm 0.073\end{array}$ \\
\hline$A B$ & $n=64$ & $\begin{aligned} & 33.50^{\mathrm{B}} \\
\pm & 24.60\end{aligned}$ & $\begin{array}{r}292.60 \\
\pm 301.10\end{array}$ & $\begin{array}{r}14.90 \\
\pm 11.12\end{array}$ & $\begin{array}{r}0.070 \\
\pm 0.229\end{array}$ & $\begin{array}{r}9.30^{\mathrm{B}} \\
\pm 7.54\end{array}$ & $\begin{array}{r}0.010 \\
\pm 0.092\end{array}$ \\
\hline $\mathrm{BB}$ & $n=54$ & $\begin{aligned} & 32.80^{\mathrm{B}} \\
\pm & 23.86\end{aligned}$ & $\begin{array}{r}285.50 \\
\pm 266.36\end{array}$ & $\begin{array}{r}15.90 \\
\pm 10.82\end{array}$ & $\begin{array}{r}0.820 \\
\pm 5.297\end{array}$ & $\begin{aligned} & 8.50^{8} \\
\pm & 7.19\end{aligned}$ & $\begin{array}{r}-0.010 \\
\pm 0.092\end{array}$ \\
\hline \multicolumn{8}{|c|}{ LALBA } \\
\hline PP & $\mathrm{n}=14$ & $\begin{aligned} & 22.90^{\mathrm{B}} \\
\pm & 23.69\end{aligned}$ & $\begin{array}{r}258.90 \\
+292.30\end{array}$ & $\begin{array}{r}8.60^{8} \\
\pm 10.40\end{array}$ & $\begin{array}{r}-0.040 \\
\pm 0.226\end{array}$ & $\begin{array}{r}7.10 \\
\pm 7.97\end{array}$ & $\begin{array}{r}-0.024 \\
\pm 0.066\end{array}$ \\
\hline PM & $n=63$ & $\begin{aligned} & 33.80^{\mathrm{AB}} \\
\pm & 24.65\end{aligned}$ & $\begin{array}{r}292.80 \\
\pm 285.77\end{array}$ & $\begin{aligned} & 15.10^{\mathrm{AB}} \\
\pm & 10.83\end{aligned}$ & $\begin{array}{r}0.680 \\
\pm 4.909\end{array}$ & $\begin{array}{r}9.30 \\
\pm 7.51\end{array}$ & $\begin{array}{r}0.004 \\
\pm 0.099\end{array}$ \\
\hline MM & $\mathrm{n}=66$ & $\begin{array}{r}40.40^{\wedge} \\
+23.99\end{array}$ & $\begin{array}{r}344.70 \\
\pm 281.43\end{array}$ & $\begin{array}{r}18.70^{\wedge} \\
\pm 10.71\end{array}$ & $\begin{array}{r}0.100 \\
\pm 0.214\end{array}$ & $\begin{array}{r}10.90 \\
\pm 7.41\end{array}$ & $\begin{array}{r}0.010 \\
\pm 0.084\end{array}$ \\
\hline & & $\begin{array}{r}35.77 \\
\pm 24.66\end{array}$ & $\begin{array}{r}313.45 \\
\pm 284.03\end{array}$ & $\begin{array}{r}16.12 \\
\pm 11.07\end{array}$ & $\begin{array}{r}0.345 \\
\pm 3.262\end{array}$ & $\begin{array}{r}9.83 \\
\pm 7.55\end{array}$ & $\begin{array}{r}0.003 \\
\pm 0.089\end{array}$ \\
\hline
\end{tabular}

$n=$ number of animals recorded, TPI - Type Production lndex $A, \mathrm{~B}-\mathrm{P} \leq 0.01$ 
TABLE 2

Means and standard deviations of PTI and PTA values of animals with different CASK/CASK-R intragenic haplotypes

\begin{tabular}{|c|c|c|c|c|c|c|}
\hline \multirow{2}{*}{$\begin{array}{l}\text { Intragenic } \\
\text { haplotype }\end{array}$} & \multicolumn{6}{|c|}{ Predicted Transmitting Ability } \\
\hline & TPI & $\begin{array}{c}\text { Milk } \\
\text { kg }\end{array}$ & $\begin{array}{c}\text { Fat } \\
\text { kg }\end{array}$ & $\begin{array}{c}\text { Fat } \\
\%\end{array}$ & $\begin{array}{l}\text { Protein } \\
\mathrm{kg}\end{array}$ & $\begin{array}{c}\text { Protein } \\
\%\end{array}$ \\
\hline CASK/CASK-R & & & & & & \\
\hline $\mathrm{AA} / \mathrm{MM} \mathrm{n}=5$ & $\begin{aligned} & 52.70^{\mathrm{A}} \\
\pm & 45.04\end{aligned}$ & $\begin{array}{r}486.40 \\
\pm 493.80\end{array}$ & $\begin{array}{r}20.50 \\
\pm 17.33\end{array}$ & $\begin{array}{r}-0.010 \\
\pm 0.222\end{array}$ & $\begin{array}{r}16.10 \\
\pm 14.22\end{array}$ & $\begin{array}{r}0.200 \\
\pm 0.053\end{array}$ \\
\hline AA/PM $n=38$ & $\begin{aligned} & 36.70^{A} \\
\pm & 26.61\end{aligned}$ & $\begin{array}{r}329.50 \\
\pm 291.54\end{array}$ & $\begin{array}{r}16.60 \\
\pm 12.40\end{array}$ & $\begin{array}{r}1.100 \\
\pm 6.320\end{array}$ & $\begin{array}{r}10.10 \\
\pm 7.79\end{array}$ & $\begin{array}{r}-0.003 \\
\pm 0.102\end{array}$ \\
\hline AA/PP $\quad n=49$ & $\begin{aligned} & 37.00^{\wedge} \\
\pm & 22.55\end{aligned}$ & $\begin{array}{r}361.50 \\
\pm 259.89\end{array}$ & $\begin{array}{r}17.00 \\
\pm 9.72\end{array}$ & $\begin{array}{r}0.050 \\
\pm 0.200\end{array}$ & $\begin{array}{r}10.00 \\
\pm 7.25\end{array}$ & $\begin{array}{r}-0.030 \\
\pm 0.066\end{array}$ \\
\hline $\mathrm{AB} / \mathrm{PM} \mathrm{n}=11$ & $\begin{aligned} & 27.80^{\mathrm{B}} \\
\pm & 29.78\end{aligned}$ & $\begin{array}{r}222.10 \\
\pm 331.83\end{array}$ & $\begin{array}{r}11.70 \\
\pm 12.67\end{array}$ & $\begin{array}{r}0.050 \\
\pm 0.144\end{array}$ & $\begin{array}{r}8.00 \\
\pm 9.09\end{array}$ & $\begin{array}{r}0.030 \\
\pm 0.078\end{array}$ \\
\hline $\mathrm{AB} / \mathrm{PP} \quad \mathrm{n}=34$ & $\begin{aligned} & 34.20^{\mathrm{AB}} \\
\pm & 21.52\end{aligned}$ & $\begin{array}{r}237.00 \\
\pm 254.53\end{array}$ & $\begin{array}{r}15.90 \\
\pm 10.01\end{array}$ & $\begin{array}{r}0.130 \\
\pm 0.198\end{array}$ & $\begin{array}{r}9.20 \\
\pm 6.35\end{array}$ & $\begin{array}{r}0.040 \\
\pm 0.098\end{array}$ \\
\hline & $\begin{array}{r}35.77 \\
\pm 24.66\end{array}$ & $\begin{array}{r}313.45 \\
\pm 284.03\end{array}$ & $\begin{array}{r}16.12 \\
\pm 11.07\end{array}$ & $\begin{array}{r}0.345 \\
\pm 3.262\end{array}$ & $\begin{array}{r}9.83 \\
\pm 7.55\end{array}$ & $\begin{array}{r}0.003 \\
\pm 0.089\end{array}$ \\
\hline
\end{tabular}

$\mathrm{n}=$ number of animals recorded, TPI - Type Production Index

$\mathrm{A} . \mathrm{B}-\mathrm{P} \leq 0.01$

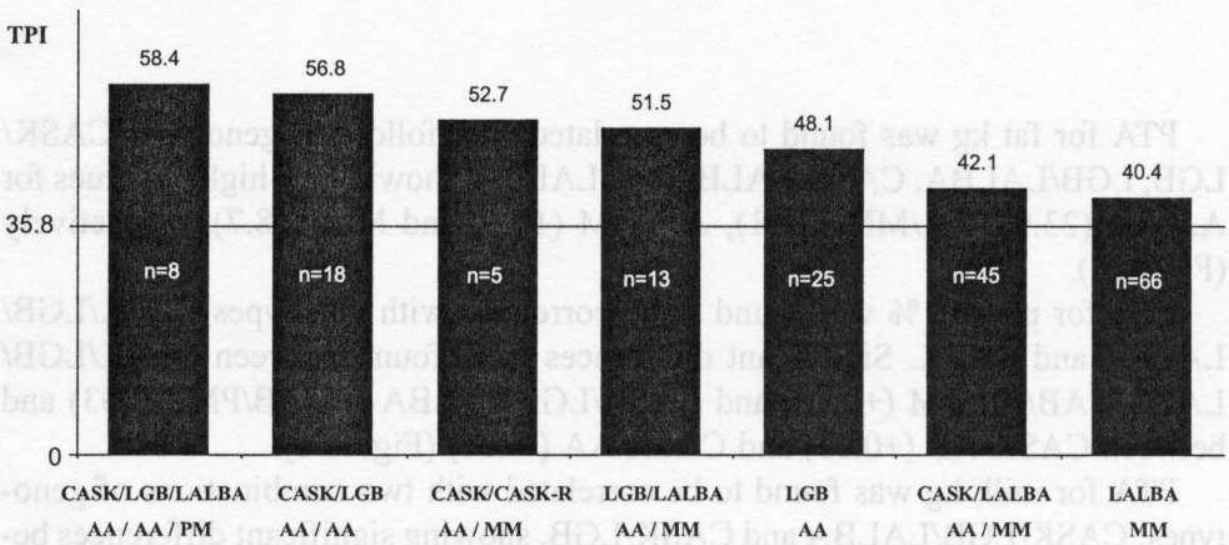

Figure 1. Genotypes, intragenic haplotypes and combination of genotypes showing the highest TPI values 
TABLE 3

Means and standard deviations of PTI and PTA valucs of animals with different combinations of genotypes CASK/LALBA

\begin{tabular}{|c|c|c|c|c|c|c|}
\hline \multirow{2}{*}{$\begin{array}{l}\text { Combination } \\
\text { of genotypes }\end{array}$} & \multirow[b]{2}{*}{ TPI } & \multicolumn{5}{|c|}{ Predicted Transmitting Ability } \\
\hline & & $\begin{array}{c}\text { Milk } \\
\text { kg }\end{array}$ & $\begin{array}{l}\text { Fat } \\
\mathrm{kg}\end{array}$ & $\begin{array}{c}\text { Fat } \\
\%\end{array}$ & $\begin{array}{c}\text { Protein } \\
\text { kg }\end{array}$ & $\begin{array}{c}\text { Protein } \\
\%\end{array}$ \\
\hline \multicolumn{7}{|l|}{ CASK/LALBA } \\
\hline \multirow[t]{2}{*}{$\mathrm{AA} / \mathrm{MM} \mathrm{n}=45$} & $42.10^{\mathrm{A}}$ & 390.60 & $19.30^{\mathrm{A}}$ & 0.080 & 11.40 & -0.010 \\
\hline & \pm 26.45 & \pm 306.13 & \pm 11.42 & \pm 0.226 & \pm 8.27 & \pm 0.078 \\
\hline \multirow[t]{2}{*}{$\mathrm{AA} / \mathrm{PM} \quad \mathrm{n}=41$} & $37.70^{\mathrm{AB}}$ & 350.60 & $16.70^{\wedge \mathrm{B}}$ & 1.000 & 10.50 & -0.010 \\
\hline & \pm 22.74 & \pm 258.99 & \pm 10.14 & \pm 6.086 & \pm 7.06 & \pm 0.096 \\
\hline \multirow[t]{2}{*}{$\mathrm{AA} / \mathrm{PP}$} & $14.50^{\mathrm{B}}$ & 150.90 & $6.90^{B}$ & 0.003 & 3.80 & -0.020 \\
\hline & \pm 22.60 & \pm 243.73 & \pm 9.67 & \pm 0.211 & \pm 7.49 & \pm 0.047 \\
\hline \multirow[t]{2}{*}{$\mathrm{AB} / \mathrm{MM} \mathrm{n}=21$} & $36.80^{\mathrm{AB}}$ & 246.60 & $17.30^{n / 3}$ & 0.160 & 9.70 & 0.050 \\
\hline & \pm 17.63 & \pm 190.77 & \pm 9.12 & \pm 0.178 & \pm 5.09 & \pm 0.086 \\
\hline \multirow[t]{2}{*}{$\mathrm{AB} / \mathrm{PM} \quad \mathrm{n}=21$} & $27.20^{\wedge \mathrm{B}}$ & 187.10 & $12.70^{\wedge \mathrm{B}}$ & 0.110 & 7.30 & 0.040 \\
\hline & \pm 27.40 & \pm 315.13 & \pm 11.69 & \pm 0.197 & \pm 8.22 & \pm 0.098 \\
\hline \multirow[t]{4}{*}{$\mathrm{AB} / \mathrm{PP}$} & $34.90^{\wedge \mathrm{B}}$ & 423.80 & $11.10^{\mathrm{AB}}$ & -0.120 & 11.90 & -0.040 \\
\hline & \pm 24.43 & \pm 340.59 & \pm 13.15 & \pm 0.273 & \pm 7.46 & \pm 0.096 \\
\hline & 35.77 & 313.45 & 16.12 & 0.345 & 9.83 & 0.003 \\
\hline & \pm 24.66 & \pm 284.03 & \pm 11.07 & \pm 3.262 & \pm 7.55 & \pm 0.089 \\
\hline
\end{tabular}

$\mathrm{n}=$ number of animals recorded, TPI - Type Production Index

A.B $-P \leq 0.01$

PTA for fat $\mathrm{kg}$ was found to be correlated with following genotypes CASK/ LGB, LGB/LALBA, CASK/LALBA and LALBA, showing the highest values for AA/AA (23.9), AA/MM (21.1), AA/MM (19.3) and MM (18.7), respectively (Figure 3).

PTA for protein \% was found to be correlated with genotypes CASK/LGB/ LALBA and CASK. Significant differences were found between CASK/LGB/ LALBA AB/AB/PM $(+0.10)$ and CASK/LGB/LALBA AA/BB/PM (-0.03) and between CASK AB (+0.03) and CASK AA (-0.01) (Figure 4).

PTA for milk $\mathrm{kg}$ was found to be corrclated with two combinations of genotypes: $\mathrm{CASK} / \mathrm{LGB} / \mathrm{LALBA}$ and $\mathrm{CASK} / \mathrm{LGB}$, showing significant differences between $\mathrm{AA} / \mathrm{AA} / \mathrm{MM}(542.1)$ and $\mathrm{AB} / \mathrm{AB} / \mathrm{PM}(17.1) \mathrm{AA} / \mathrm{AA}(513.2)$ and $\mathrm{AB} / \mathrm{AA}$ (217.2), respectively (Figure 5). 


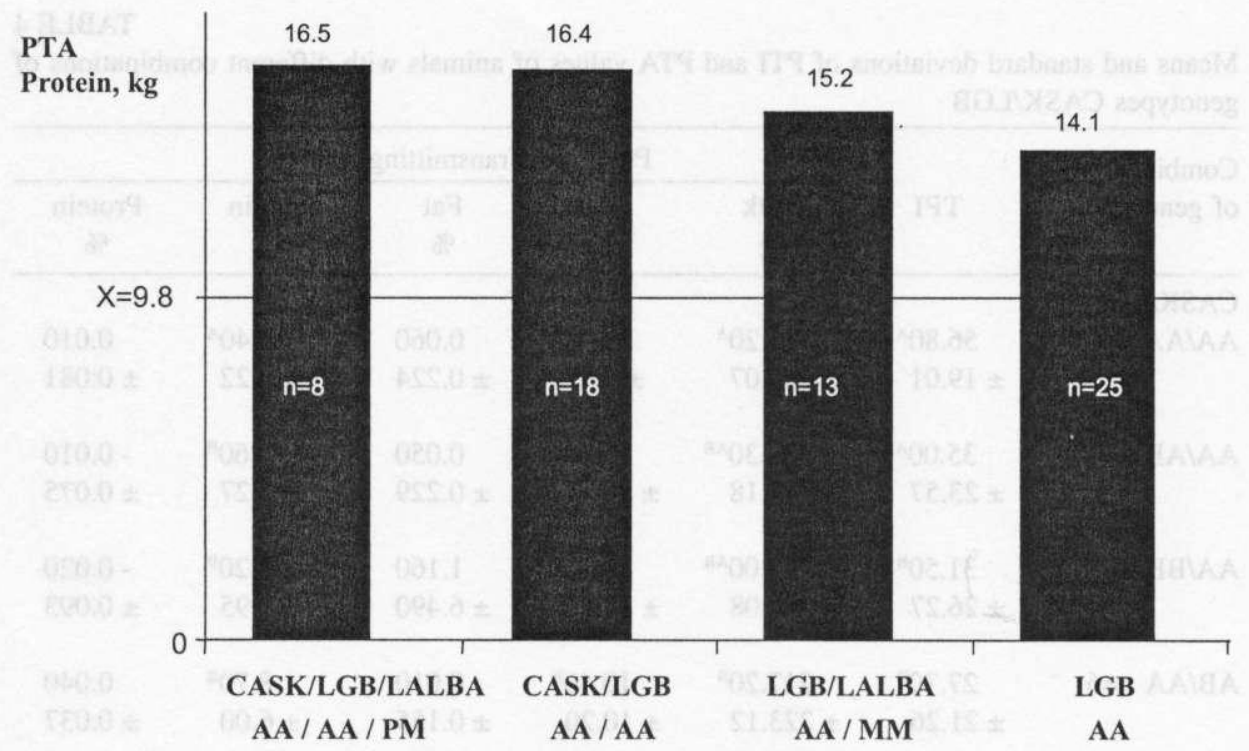

Figure 2. Genotypes and combination of genotypes showing the highest PTA for protein $\mathrm{kg}$

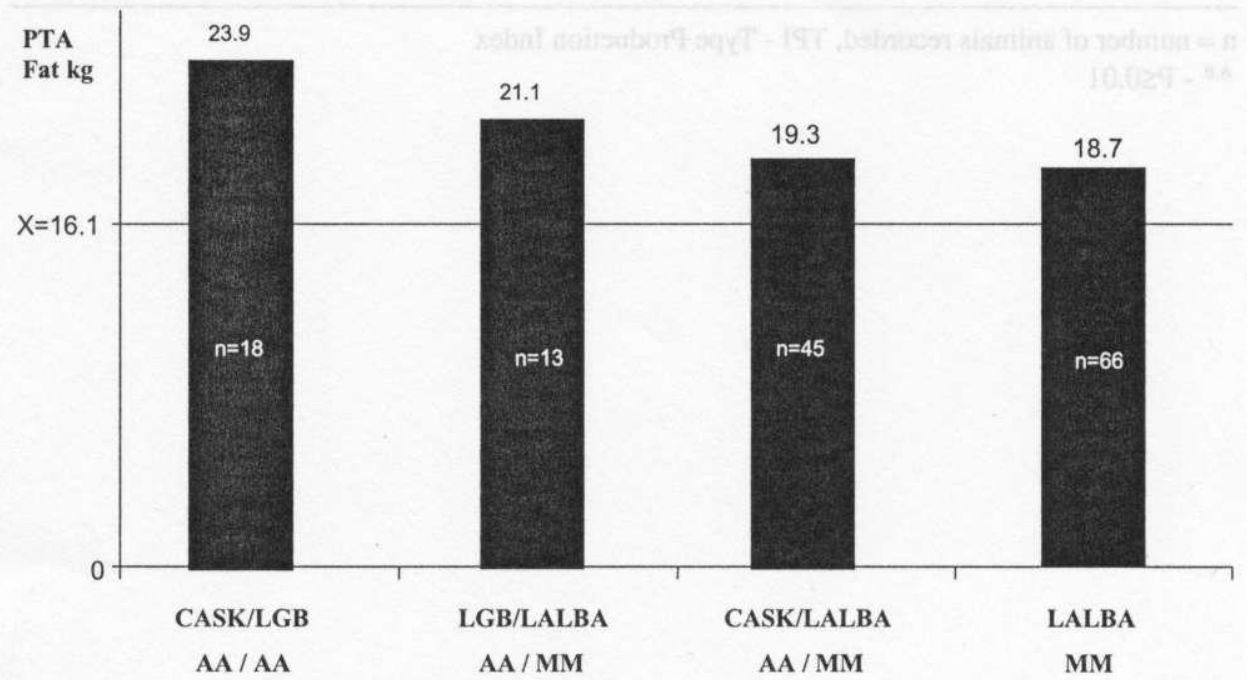

Figure 3. Genotypes and combination of genotypes showing the highest PTA for fat $\mathrm{kg}$ 
TABLE 4

Means and standard deviations of PTI and PTA values of animals with different combinations of genotypes CASK/LGB

\begin{tabular}{|c|c|c|c|c|c|c|}
\hline \multirow{2}{*}{$\begin{array}{l}\text { Combination } \\
\text { of genotypes }\end{array}$} & \multirow[b]{2}{*}{ TPI } & \multicolumn{4}{|c|}{ Predicted Transmitting Ability } & \multirow[b]{2}{*}{$\begin{array}{c}\text { Protein } \\
\%\end{array}$} \\
\hline & & $\begin{array}{l}\text { Milk } \\
\text { kg }\end{array}$ & $\begin{array}{l}\text { Fat } \\
\text { kg }\end{array}$ & $\begin{array}{c}\text { Fat } \\
\%\end{array}$ & $\begin{array}{c}\text { Protein } \\
\mathrm{kg}\end{array}$ & \\
\hline CASK/LGB & & & & & & \\
\hline AA/AA $n=18$ & $\begin{array}{r}56.80^{A} \\
\pm 19.01\end{array}$ & $\begin{aligned} & 513.20^{\wedge} \\
\pm & 225.07\end{aligned}$ & $\begin{array}{r}23.90^{A} \\
\pm 8.67\end{array}$ & $\begin{array}{r}0.060 \\
\pm 0.224\end{array}$ & $\begin{array}{l}16.40^{A} \\
\pm 6.22\end{array}$ & $\begin{array}{r}0.010 \\
\pm 0.081\end{array}$ \\
\hline $\mathrm{AA} / \mathrm{AB} \mathrm{n}=40$ & $\begin{aligned} & 35.00^{\mathrm{AB}} \\
\pm & 23.57\end{aligned}$ & $\begin{aligned} & 334.30^{\mathrm{AB}} \\
\pm & 289.18\end{aligned}$ & $\begin{aligned} & 15.80^{\mathrm{AB}} \\
\pm & 10.74\end{aligned}$ & $\begin{array}{r}0.050 \\
\pm 0.229\end{array}$ & $\begin{aligned} & 9.60^{\mathrm{B}} \\
\pm & 7.27\end{aligned}$ & $\begin{array}{r}-0.010 \\
\pm 0.075\end{array}$ \\
\hline $\mathrm{AA} / \mathrm{BB} \mathrm{n}=36$ & $\begin{aligned} & 31.50^{\mathrm{B}} \\
\pm & 26.27\end{aligned}$ & $\begin{aligned} & 293.00^{\mathrm{AB}} \\
\pm & 287.08\end{aligned}$ & $\begin{aligned} & 15.20^{\mathrm{AB}} \\
\pm & 11.64\end{aligned}$ & $\begin{array}{r}1.160 \\
\pm 6.490\end{array}$ & $\begin{array}{r}8.20^{\mathrm{B}} \\
\pm 7.95\end{array}$ & $\begin{array}{l}-0.020 \\
\pm 0.093\end{array}$ \\
\hline $\mathrm{AB} / \mathrm{AA} \mathrm{n}=6$ & $\begin{aligned} & 27.70^{\mathrm{B}} \\
\pm & 21.26\end{aligned}$ & $\begin{aligned} & 217.20^{\mathrm{B}} \\
\pm & 223.12\end{aligned}$ & $\begin{aligned} & 10.40^{\mathrm{B}} \\
\pm & 10.20\end{aligned}$ & $\begin{array}{r}0.010 \\
\pm 0.155\end{array}$ & $\begin{aligned} & 8.70^{\mathrm{B}} \\
\pm & 6.00\end{aligned}$ & $\begin{array}{r}0.040 \\
\pm 0.037\end{array}$ \\
\hline $\mathrm{AB} / \mathrm{AB} \mathrm{n}=24$ & $\begin{aligned} & 30.90^{\mathrm{B}} \\
\pm & 26.55\end{aligned}$ & $\begin{aligned} & 223.00^{\mathrm{B}} \\
\pm & 313.79\end{aligned}$ & $\begin{aligned} & 13.40^{\mathrm{AB}} \\
\pm & 11.81\end{aligned}$ & $\begin{array}{r}0.100 \\
\pm 0.230\end{array}$ & $\begin{aligned} & 8.70^{\mathrm{B}} \\
\pm & 8.10\end{aligned}$ & $\begin{array}{r}0.040 \\
\pm 0.108\end{array}$ \\
\hline $\mathrm{AB} / \mathrm{BB} \mathrm{n}=17$ & $\begin{aligned} & 35.90^{\mathrm{AB}} \\
\pm & 18.98\end{aligned}$ & $\begin{aligned} & 268.90^{\text {АВ }} \\
\pm & 233.03\end{aligned}$ & $\begin{array}{l}17.70^{\mathrm{AB}} \\
\pm 9.22\end{array}$ & $\begin{array}{r}0.150 \\
\pm 0.193\end{array}$ & $\begin{aligned} & 9.10^{\mathrm{B}} \\
\pm & 5.70\end{aligned}$ & $\begin{array}{r}0.010 \\
\pm 0.088\end{array}$ \\
\hline & $\begin{array}{r}35.77 \\
\pm 24.66\end{array}$ & $\begin{array}{r}313.45 \\
\pm 284.03\end{array}$ & $\begin{array}{r}16.12 \\
\pm 11.07\end{array}$ & $\begin{array}{r}0.345 \\
\pm 3.262\end{array}$ & $\begin{array}{r}9.83 \\
\pm 7.55\end{array}$ & $\begin{array}{r}0.003 \\
\pm 0.089\end{array}$ \\
\hline
\end{tabular}

$\mathrm{n}=$ number of animals recorded, TPI - Type Production Index $\mathrm{A}, \mathrm{B}-\mathrm{P} \leq 0.01$ 
TABLE 5

Means and standard deviations of PTI and PTA values of animals with different combinations of LGB/LALBA genotypes

\begin{tabular}{|c|c|c|c|c|c|c|}
\hline \multirow{2}{*}{$\begin{array}{l}\text { Combination } \\
\text { of genotypes }\end{array}$} & \multicolumn{6}{|c|}{ Predicted Transmitting Ability } \\
\hline & TPI & $\begin{array}{c}\text { Milk } \\
\text { kg }\end{array}$ & $\begin{array}{l}\text { Fat } \\
\mathrm{kg}\end{array}$ & $\begin{array}{c}\text { Fat } \\
\%\end{array}$ & $\begin{array}{l}\text { Protein } \\
\text { kg }\end{array}$ & $\begin{array}{c}\text { Protein } \\
\%\end{array}$ \\
\hline \multicolumn{7}{|l|}{ LGB/LALBA } \\
\hline \multirow{2}{*}{$\mathrm{AA} / \mathrm{MM} \mathrm{n}=13$} & $51.50^{A}$ & 488.80 & $21.10^{A}$ & 0.020 & $15.20^{A}$ & -0.002 \\
\hline & \pm 20.66 & \pm 224.09 & \pm 10.24 & \pm 0.244 & \pm 6.25 & \pm 0.064 \\
\hline \multirow[t]{2}{*}{$\mathrm{AA} / \mathrm{PM} \mathrm{n}=12$} & $44.30^{\wedge}$ & 360.80 & $18.30^{\mathrm{A}}$ & 0.060 & $13.00^{\mathrm{A}}$ & 0.040 \\
\hline & \pm 27.07 & \pm 283.56 & \pm 12.35 & \pm 0.157 & \pm 7.87 & \pm 0.078 \\
\hline \multirow[t]{2}{*}{$\mathrm{AB} / \mathrm{MM} \mathrm{n}=36$} & $37.80^{\mathrm{A}}$ & 317.60 & $17.70^{\mathrm{A}}$ & 0.100 & $10.00^{\mathrm{AB}}$ & 0.010 \\
\hline & \pm 23.56 & \pm 288.31 & \pm 10.83 & \pm 0.190 & \pm 6.97 & \pm 0.076 \\
\hline \multirow[t]{2}{*}{$\mathrm{AB} / \mathrm{PM} n=19$} & $\quad 26.60^{A B}$ & 218.30 & $11.10^{\mathrm{AB}}$ & 0.050 & $7.70^{\mathrm{AB}}$ & 0.030 \\
\hline & \pm 26.24 & \pm 317.05 & \pm 10.52 & \pm 0.260 & \pm 8.34 & \pm 0.122 \\
\hline \multirow[t]{2}{*}{$\mathrm{AB} / \mathrm{PP} \quad \mathrm{n}=9$} & $30.80^{\mathrm{AB}}$ & 349.10 & $11.60^{\mathrm{AB}}$ & -0.050 & $9.60^{\mathrm{AB}}$ & -0.030 \\
\hline & \pm 24.19 & \pm 323.43 & \pm 11.36 & \pm 0.284 & \pm 8.39 & \pm 0.075 \\
\hline \multirow[t]{2}{*}{$\mathrm{BB} / \mathrm{MM} \mathrm{n}=17$} & $37.60^{\mathrm{A}}$ & 292.10 & $18.70^{\mathrm{A}}$ & 0.170 & $9.50^{\mathrm{AB}}$ & 0.010 \\
\hline & \pm 26.07 & \pm 284.28 & \pm 11.14 & \pm 0.226 & \pm 8.27 & \pm 0.114 \\
\hline \multirow[t]{2}{*}{$\mathrm{BB} / \mathrm{PM} \quad \mathrm{n}=32$} & $34.10^{\mathrm{AB}}$ & 311.50 & $16.30^{\wedge}$ & 1.290 & $8.90^{\mathrm{AB}}$ & -0.020 \\
\hline & \pm 21.91 & \pm 266.24 & \pm 10.05 & \pm 6.880 & \pm 6.60 & \pm 0.084 \\
\hline \multirow[t]{2}{*}{$\mathrm{BB} / \mathrm{PP} \quad \mathrm{n}=5$} & $8.50^{\mathrm{B}}$ & 96.60 & $3.40^{\mathrm{B}}$ & -0.020 & $2.60^{\mathrm{B}}$ & -0.010 \\
\hline & \pm 15.97 & \pm 131.30 & \pm 6.23 & \pm 0.060 & \pm 6.06 & \pm 0.048 \\
\hline & 35.77 & 313.45 & 16.12 & 0.345 & 9.83 & 0.003 \\
\hline & \pm 24.66 & \pm 284.03 & \pm 11.07 & \pm 3.262 & \pm 7.55 & \pm 0.089 \\
\hline
\end{tabular}

$\mathrm{n}=$ number of animals recorded, TPI - Type Production Index $\mathrm{A}, \mathrm{B}-\mathrm{P} \leq 0.01$ 
TABLE 6

Means and standard deviations of PTI and PTA values of animals with different CASK/LGB/L.AL.BA combinations of genotypes

\begin{tabular}{|c|c|c|c|c|c|c|c|}
\hline \multirow{2}{*}{\multicolumn{2}{|c|}{$\begin{array}{l}\text { Combination } \\
\text { of genotypes }\end{array}$}} & \multirow[b]{2}{*}{ TPl } & \multicolumn{4}{|c|}{ Predicted Transmitting Ability } & \multirow[b]{2}{*}{$\begin{array}{c}\text { Protein } \\
\%\end{array}$} \\
\hline & & & $\begin{array}{l}\text { Milk } \\
\mathrm{kg}\end{array}$ & $\begin{array}{l}\text { Fat } \\
\mathrm{kg}\end{array}$ & $\begin{array}{l}\text { Fat } \\
\%\end{array}$ & $\begin{array}{c}\text { Protein } \\
\mathrm{kg}\end{array}$ & \\
\hline \multicolumn{8}{|c|}{ CASK/LGB/LALBA } \\
\hline \multirow[t]{2}{*}{$\mathrm{AA} / \mathrm{AA} / \mathrm{MM}$} & $n=10$ & $55.60^{\mathrm{AB}}$ & $542.10^{\wedge}$ & 22.70 & 0.010 & $16.40^{\mathrm{A}}$ & $-0.010^{\lambda \mathrm{B}}$ \\
\hline & & \pm 20.86 & \pm 223.43 & \pm 10.50 & \pm 0.277 & \pm 6.44 & \pm 0.071 \\
\hline \multirow[t]{2}{*}{$\mathrm{AA} / \mathrm{AA} / \mathrm{PM}$} & $11=8$ & $58.40^{\prime}$ & $477.10^{\wedge}$ & 25.50 & 0.110 & $16.50^{\mathrm{A}}$ & $0.030^{\wedge \mathrm{B}}$ \\
\hline & & \pm 17.71 & \pm 236.99 & \pm 5.98 & \pm 0.131 & \pm 6.37 & \pm 0.092 \\
\hline \multirow[t]{2}{*}{$\mathrm{AA} / \mathrm{AB} / \mathrm{MM}$} & $\mathrm{n}=23$ & $39.80^{\mathrm{ABC}}$ & $355.70^{\mathrm{AR}}$ & 19.20 & 0.100 & $10.30^{\mathrm{AB}}$ & $-0.010^{413}$ \\
\hline & & \pm 25.63 & \pm 314.94 & \pm 11.44 & \pm 0.209 & \pm 7.76 & \pm 0.065 \\
\hline \multirow[t]{2}{*}{$\mathrm{AA} / \mathrm{AB} / \mathrm{PM}$} & $\mathrm{n}=12$ & $30.30^{\wedge B C}$ & $335.70^{\mathrm{Ab}}$ & 11.00 & -0.006 & $9.60^{A B}$ & $-0.020^{\mathrm{AB}}$ \\
\hline & & \pm 18.43 & \pm 251.43 & \pm 7.63 & $\pm 0.23 !$ & \pm 6.01 & \pm 0.103 \\
\hline \multirow[t]{2}{*}{$\mathrm{AA} / \mathrm{AB} / \mathrm{PP}$} & $n=5$ & $24.50^{\mathrm{BC}}$ & $232.20^{\mathrm{AB}}$ & 11.60 & 0.040 & $6.50^{\mathrm{AB}}$ & $-0.020^{\mathrm{AB}}$ \\
\hline & & \pm 23.07 & \pm 283.37 & \pm 9.31 & \pm 0.270 & \pm 8.39 & \pm 0.048 \\
\hline \multirow[t]{2}{*}{$\mathrm{AA} / \mathrm{BB} / \mathrm{MM}$} & $n=12$ & $35.50^{\mathrm{ABC}}$ & $331.10^{A B}$ & 16.50 & 0.090 & $9.50^{\mathrm{AB}}$ & $-0.020^{\mathrm{AB}}$ \\
\hline & & \pm 30.08 & \pm 329.19 & \pm 12.29 & \pm 0.221 & \pm 9.49 & \pm 0.107 \\
\hline \multirow[t]{2}{*}{$\mathrm{AA} / \mathrm{BB} / \mathrm{PM}$} & $n=21$ & $34.00^{\mathrm{ARC}}$ & $310.90^{\mathrm{AB}}$ & 16.70 & 1.940 & $8.70^{\wedge B}$ & $-0.030^{\mathrm{A}}$ \\
\hline & & \pm 22.76 & \pm 267.46 & \pm 10.53 & \pm 8.493 & \pm 6.89 & \pm 0.092 \\
\hline \multirow[t]{2}{*}{$\mathrm{AB} / \mathrm{AB} / \mathrm{MM}$} & $n=1.3$ & $34.20^{\mathrm{ABC}}$ & $250.20^{\mathrm{AB}}$ & 15.10 & 0.100 & $9.50^{A B}$ & $0.040^{\mathrm{AB}}$ \\
\hline & & \pm 19.81 & \pm 229.96 & \pm 9.53 & \pm 0.156 & \pm 5.59 & \pm 0.084 \\
\hline \multirow[t]{2}{*}{$\mathrm{AB} / \mathrm{AB} / \mathrm{PM}$} & $n=7$ & $20.20^{\mathrm{C}}$ & $17.10^{\mathrm{s}}$ & 11.40 & 0.240 & $4.40^{\mathrm{B}}$ & $0.100^{\mathrm{B}}$ \\
\hline & & \pm 36.99 & \pm 333.02 & \pm 15.00 & \pm 0.202 & \pm 11.07 & \pm 0.121 \\
\hline \multirow[t]{2}{*}{$\mathrm{AB} / \mathrm{BB} / \mathrm{MM}$} & $\mathrm{n}=5$ & $42.80^{\mathrm{ABC}}$ & $198.60^{\mathrm{AH}}$ & 23.90 & 0.370 & $9.40^{\mathrm{AB}}$ & $0.070^{k+3}$ \\
\hline & & \pm 13.49 & \pm 98.88 & \pm 5.81 & \pm 0.067 & \pm 5.12 & \pm 0.120 \\
\hline \multirow{4}{*}{\multicolumn{2}{|c|}{$\mathrm{AB} / \mathrm{BB} / \mathrm{PM}$}} & $34.30^{A B C}$ & $312.70^{\mathrm{AB}}$ & 15.60 & 0.060 & $9.30^{\mathrm{AB}}$ & $-0.010^{A B}$ \\
\hline & & \pm 21.24 & \pm 276.90 & \pm 9.53 & \pm 0.156 & \pm 6.32 & \pm 0.066 \\
\hline & & 35.77 & 313.45 & 16.12 & 0.345 & 9.83 & 0.003 \\
\hline & & \pm 24.66 & \pm 284.03 & \pm 11.07 & \pm 3.262 & \pm 7.55 & \pm 0.089 \\
\hline
\end{tabular}

$\mathrm{n}=$ number of animals recorded, TPI - Type Production Index A.B $-P \leq 0.01$ 


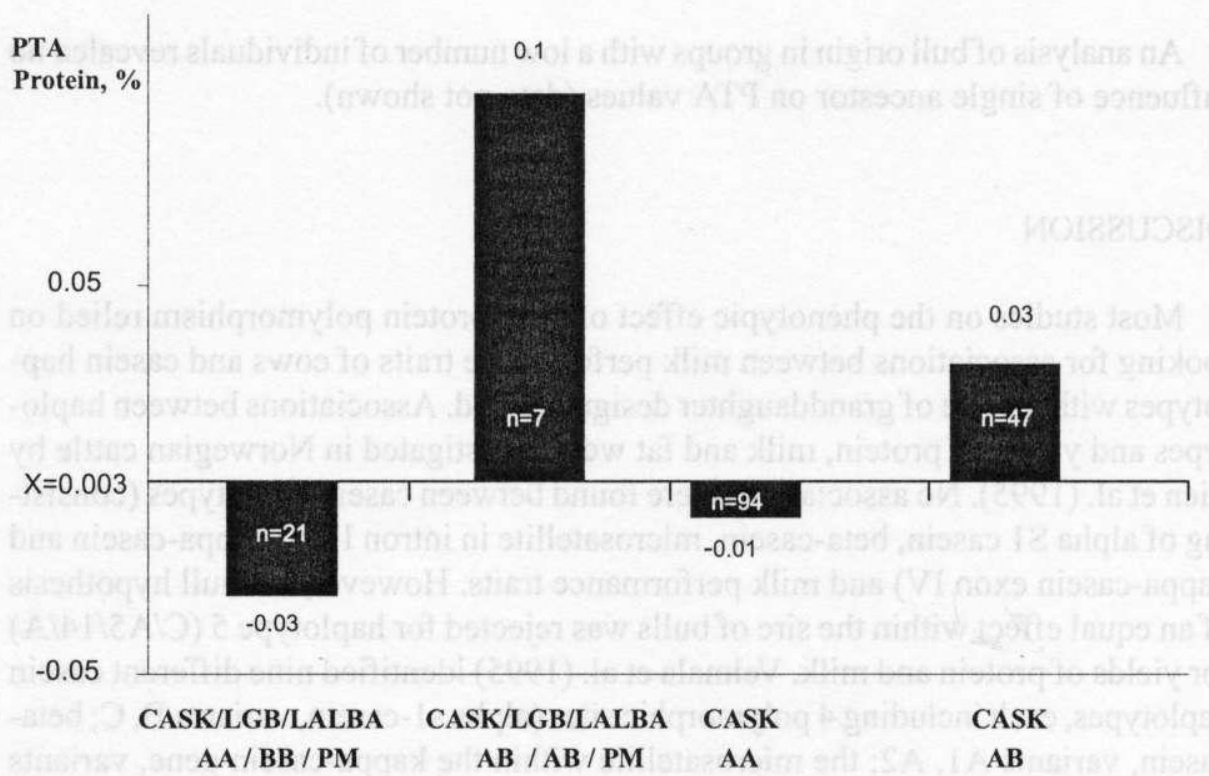

Figure 4. Genotypes and combination of genotypes showing the highest and the lowest PTA for protein $\%$

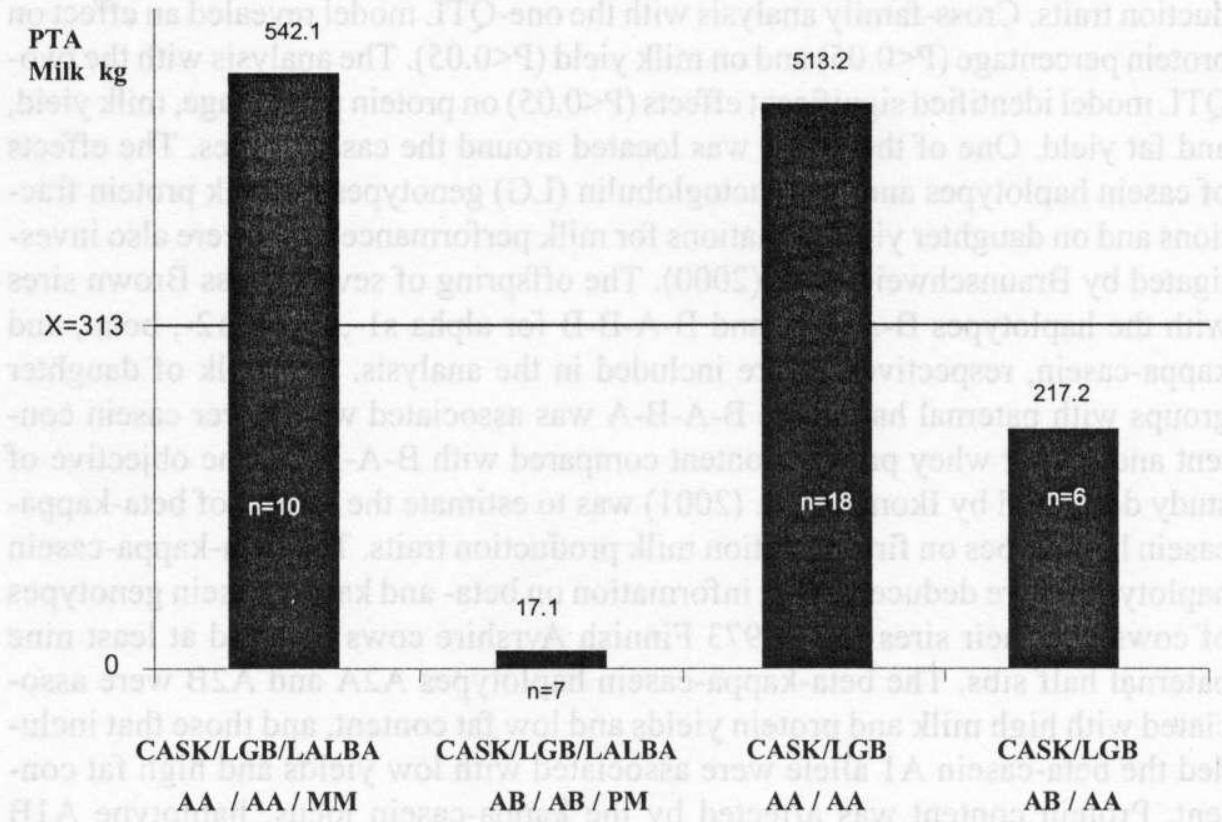

Figure 5. Combination of genotypes showing the highest and the lowest PTA for milk kg 
An analysis of bull origin in groups with a low number of individuals revealed no influence of single ancestor on PTA values (data not shown).

\section{DISCUSSION}

Most studies on the phenotypic effect of milk protein polymorphism relied on looking for associations between milk performance traits of cows and casein haplotypes with the use of granddaughter design method. Associations between haplotypes and yields of protein, milk and fat were investigated in Norwegian cattle by Lien et al. (1995). No associations were found between casein haplotypes (consisting of alpha S1 casein, beta-casein, microsatellite in intron III of kappa-casein and kappa-casein exon IV) and milk performance traits. However, the null hypothesis of an equal effect within the sire of bulls was rejected for haplotype 5 (C/A5/14/A) for yields of protein and milk. Velmala et al. (1995) identified nine different casein haplotypes, each including 4 polymorphic sites (alpha s l-casein, variants $\mathrm{B}, \mathrm{C}$; betacasein, variants A1, A2; the microsatellite within the kappa-casein gene, variants ms5, ms4 and kappa-casein exon IV, variants $A, B, E$ ) but no direct effect of the casein haplotypes on the milk performance traits was found. In a later study, Velmala et al. (1999) scanned chromosome 6 with 11 markers, ten microsatellites and casein haplotype to identify quantitative trait loci (QTLs) affecting some milk production traits. Cross-family analysis with the one-QTL model revealed an effect on protein percentage $(\mathrm{P}<0.05)$ and on milk yield $(\mathrm{P}<0.05)$. The analysis with the twoQTL model identified significant effects $(P<0.05)$ on protein percentage, milk yield, and fat yield. One of the QTLs was located around the casein genes. The effects of casein haplotypes and beta-lactoglobulin (LG) genotypes on milk protein fractions and on daughter yield deviations for milk performance traits were also investigated by Braunschweig et al. (2000). The offspring of seven Swiss Brown sires with the haplotypes B-A-B-A and B-A-B-B for alpha s1-, alpha s 2-, beta-, and kappa-casein, respectively, were included in the analysis. The milk of daughter groups with paternal haplotype B-A-B-A was associated with lower casein content and higher whey protein content compared with B-A-B-B. The objective of study described by Ikonen et al. (2001) was to estimate the effects of beta-kappacasein haplotypes on first-lactation milk production traits. The beta-kappa-casein haplotypes were deduced using information on beta- and kappa-casein genotypes of cows and their sires for 16,973 Finnish Ayrshire cows that had at least nine paternal half sibs. The beta-kappa-casein haplotypes $\mathrm{A} 2 \mathrm{~A}$ and $\mathrm{A} 2 \mathrm{~B}$ were associated with high milk and protein yields and low fat content, and those that included the beta-casein Al allele were associated with low yields and high fat content. Protein content was affected by the kappa-casein locus; haplotype A1B was associated with high protein content and $\mathrm{AlE}$ was with low protein content. 
The haplotype combination $\mathrm{A} 1 \mathrm{~A}+\mathrm{A} 2 \mathrm{~B}$ was associated with $140 \mathrm{~kg}$ more milk yield $(P<0.045)$ and 0.03 percentage units less protein content $(P<0.055)$ than combination $\mathrm{A} 1 \mathrm{~B}+\mathrm{A} 2 \mathrm{~A}$, and combination $\mathrm{A} 1 \mathrm{~A}+\mathrm{A} 2 \mathrm{E}$ showed 0.02 percentage units greater protein content $(\mathrm{P}<0.098)$ than $\mathrm{A} 1 \mathrm{E}+\mathrm{A} 2 \mathrm{~A}$. Lately, Nadesalingen et al. (2001) searched for associations between genotypes at a number of microsatellite loci spanning chromosomes 1 and 6 and some milk performance traits. Cross-sire analyses indicated a significant QTL for fat and protein yield, and fat percentage on chromosome 1 , and QTL effects on milk yield and protein percentage that might represent one or two QTL on chromosome 6. All these studies cited above strongly support the hypothesis that milk protein genes, especially casein genes or genes linked to the casein loci, contribute to the variation in milk yield and protein content. However, our results cannot be directly compared with this analysis because of substantial differences in statistical methodology as well as in number and type of polymorphic sites analysed. First of all, we used PTA and TPI values as a data for statistical analysis. These are a phenotypic abstract of milk performance traits generated within statistical evaluations of bull breeding values. TPI and PTA values are very convenient for statistical analysis, because they are highly processed data in which most of the non-genetic influences are excluded and represent the relative advantage in milk performance traits of certain bulls in comparison to the mean of all other bulls evaluated. TPI and PTA are dynamic values being continuously corrected by the input of new data of the bulls' progeny and relatives' performance.

Secondly, the number of loci functionally engaged in milk protein synthesis was extended by the addition of polymorphic sites located in regulatory sequences of milk protein genes. It was considered that all milk protein genes are co-ordinately expressed in the epithelial cells of the mammary gland and therefore these genes should be taken altogether as a source of variability on milk protein content and yield. Therefore, 6 polymorphic sites were selected encompassing the whole casein linkage group (CSN1S1 located on $5^{\circ}$ end and CASK-R and CASK located on the 3' end), and whey protein genes (LGB and LALBA). To avoid the effect of one ancestor on a breeding values of bulls with particular genotype or haplotype the relatedness of bulls was analysed (data not shown). The analysis revealed that $70-100 \%$ of the bulls in certain genotype/haplotype group have different fathers which fulfils the object's independence criterion required in the one-factor variance analysis.

In using this approach, many associations were found between single genotypes (Table 1), intragenic haplotypes CASK/CASK-R (Table 2) and some combinations of genotypes (Tables $3,4,5,6$ ) and TPI and PTA values. Some associations are very similar to those obtained for milk performance traits of cows, e.g., namely CASK AB and LGB AA genotypes superior for protein percentage (Jakob and Puhan, 1992). 
The importance of milk protein intragenic haplotypes were the subject of previous studies conducted on cow populations. Statistically significant differences $(\mathrm{P}<0.01)$ for milk protein percentage were found. The highest protein percentage was found for cows with the CASK/CASK-R AA/PM intragenic haplotype (Kamiński, 2000). In the current study, CASK/CASK AA/MM intragenic haplotype turned out to be a marker for maximum TPI value.

The combinations of genotypes including LALBA, CASK or/and LGB showed associations with TPI and PTA values and indicated the general rule: the more chromosomes included in the analysis, the more the associations with polygenic traits. Especially, the triple combination of genotypes CASK/LGB/LALBA showed associations with the highest TPI value and the most preferable PTA values for milk $\mathrm{kg}$, protein $\mathrm{kg}$ and protein $\%$.

Quite clearly, LALBA turned out to be very effective marker showing relationships as a single locus with TPI, PTA fat $\mathrm{kg}$ and as a member of three combinations of genotypes (Tables 4, 6 and 7). It confirms the results of Bleck and Bremel (1993b) who found mutation +15 to be associated with the some milk composition traits. Voelker et al. (1997) showed that the mutation in position -1689 (which was identified and designated by us as LALBA) is completely linked with A allele of mutation +15 identified by Bleck and Bremel (1993a).

The results obtained in this analysis should be applied to a larger population of bulls representing all possible genotypes, haplotypes and combinations of genotypes along with a more effective statistical method.

\section{CONCLUSIONS}

In this report, the relationships between different genotypes, intragenic haplotypes and combinations of genotypes of six polymorphic sites within 4 milk protein genes and Type Production Index and Predicted Transmitting Abilities of Polish Black-and-White bulls is shown. Among the many theoretically possible genotypes and haplotypes, statistically significant $(\mathrm{P}<0.01)$ associations with PTI and PTA values were found between different genotypes of CASK, LGB, LALBA, intragenic haplotypes CASK/CASK-R, combinations of genotypes CASK/LALBA, CASK/LGB, LGB/LALBA and CASK/LGB/LALBA. Within these groups, the highest TPI was found for bulls carrying the following genotypes $\mathrm{CASK} / \mathrm{LGB} /$ LALBA AA/AA/PM, CASK/LGB AA/AA, CASK/CASK-R AA/MM, LGB/ LALBA AA/MM, LGB AA, CASK/LALBA AA/MM and :LALBA MM. The results confirm the occurrence QTL for milk performance traits, especially protein $\%$ on chromosome 6 and the utility of milk protein genes (mostly casein haplotypes) as genetic markers. 


\section{ACKNOWLEDGEMENTS}

The authors are thankful to Professor Krzysztof Walawski for critical review of manuscript and substantial remarks and to Stanisław Gniot (Station of Breeding and Insemination in Bydgoszcz, Division Olecko, Poland) for help in collecting the data on TPI and PTA of bulls.

\section{REFERENCES}

Bleck G.T., Bremel R.D., 1993a. Sequence and single-base polymorphisms of the bovine alphalactalbumin 5'-flanking region. Gene 126, 213-218

Bleck G.T., Bremel R.D., 1993b. Correlation of the alpha-lactalbumin $(+15)$ polymorphism to milk production and milk composition of Holstein. J. Dairy Sci. 76, 2292-2298

Bovenhuis H., Weller J.I., 1994. Mapping and analysis of dairy cattle quantitative trait loci by maximum likelihood methodology using milk protcin genes as genetic markers. Genetics 137 , $267-280$

Braunschweig M., Hagger C., Stranzinger G., Puhan Z., 2000. Associations between casein haplotypes and milk production traits of Swiss Brown cattle. J. Dairy Sci. 83, 1387-1395

Eggen A., Fries R., 1995. An integrated cytogenetic and meiotic map of the bovine genome. Anim. Genet. 26, 215-236

Ferretti L., Leone P., Sgaramella V., 1990. Long range restriction analysis of the bovine casein genes. Nucl. Acid. Res. 18, 6829-6833

Grosclaude F., Mahe M.F., Mercier J.C., Ribadeau-Dumas B., 1972. Localisation des subsitutions d'acides amines differenciant les variants $A$ et B de la caséine k bovine. Ann. Genet. Select. Anim. $4,515-521$

Hayes H.C., Petit E.J., 1993. Mapping of the b-lactoglobulin gene and an immunoglobulin $M$ heavy chain-like sequence to homologous cattle, sheep and goat chromosomes. Mamm. Genome 4, $207-$ 210

Hayes H.C., Popescu P., Dutrillaux B., 1993. Comparative gene mapping of lactoperoxidase, retinoblastoma, and alpha-lactalbumin genes in cattle, sheep, and goats. Mamm. Genome 4, 593-597

Ikonen T., Bovenhuis H., Ojala M., Routtinen O., Georges M., 2001. Associations between casein haplotypes and first lactation milk production traits in Finnish Ayrshirc cows. J. Dairy Sci. 84, $507-514$

Jakob E., Puhan Z.. 1992. Technological properties of milk as intluenced by genetic polymorphism of milk proteins (a revicw). Int. Dairy J. 2, 157-178

Kamiński S., 1996. Dde I RFLP at the 5 region of bovine kappa-casein gene. J. Appl. Genet. 37, 173178

Kamiński S., 1998. Identification of bovine kappa-casein genotypes by PCR-SSCP method. Anim. Sci. Pap. Rep. 16, 13-17

Kamiński S., 1999. Identification of the Sdu I site polymorphism within 5 flanking region of bovine alpha-lactalbumin gene. Anim. Sci. Pap. Rep. 17, 23-27

Kamiński S., 2000. Association between polymorphism within regulatory and coding fragments of bovine kappa-casein gene and milk performance traits. J. Anim. Feed Sci. 9, 435-446 
Kamiński S., Józefowicz S., 2000. SSCP polymorphism within a promoter of the bovine alfa S1 casein gene. J. Anim. Feed Sci. 9, 73-79

Kamiński S., Zabolewicz T., 1997. Genotyping of bovine beta-lactoglobulin (LGB) by PCR-SSCP technique. J. Appl. Genet. 38, 471-476

Kamiński S., Zabolewicz T., 1998. SSCP polymorphism within 5 ' region of bovine beta-lactoglobulin (LGB) gene. J. Appl. Genet. 39, 97-102

Lien S., Gomez-Raya L., Steine T., Fimland E., Rogne S., 1995. Associations between casein haplotypes and milk yield traits. J. Dairy Sci. 78, 2047-2056

Lien S., Kamiński S., Alestrom P., Rogne S., 1993. A simple and powerful method for linkage analysis by amplification of DNA from single sperm cells. Genomics 16, 4l-44

Mao I.L., Bittazzoni L.G., Aleandri R., 1992. Effects of polymorphic milk protein genes on milk yield and composition traits in Holstein cattle. Acta Agr. Scand., Sect. A, Anim. Sci. 42, 1-8

Nadesalingen J., Plante Y., Gibson J.P., 2001. Detection of QTL for milk production on chromosomes 1 and 6 of Holstein cattle. Mamm. Genome 12, 27-31

$\mathrm{Ng}-\mathrm{Kwai}$-Hang K.F., 1997. A review of the relationship between milk protein polymorphism and milk composition/milk production. In: Milk Protein Polymorphism. Proceedings of the International Dairy Federation Seminar, Palmerston. IDF Brussels, pp. 22-38

Rijnkels M., Kooiman P.M., De Boer H.A., Pieper F.R., 1997. Organisation of the bovine casein gene locus. Mamm. Genome 8, 148-152

Statsoft Inc., 1995. Statistica for Windows. Computer Program Manual

Threadgill D.W., Womack J. E., 1990. Genomic analysis of the major bovine milk protein genes. Nucl. Acid. Res. 18, 6935-6942

Velmala R., Vilkki J., Elo K., De Koning D.J., Maki-Tanila A., 1999. A search for quantitative trait loci for milk production traits on chromosome 6 in Finnish Ayrshire cattle. Anim Genet. 30, 136-143

Velmala R., Vilkki J., Elo K., Maki-Tanila A., 1995. Casein haplotypes and their association with milk production traits in the Finnish Ayrshire cattle. Anim Genet. 26, 419-425

Voelker G.R., Bleck G.T., Whecler M.B., 1997. Single-base polymorphisms within the 5' flanking region of the bovine alpha-lactalbumin gene. J. Dairy Sci. 80, 194-197

Walawski K., Sowiński G., Czarnik U., Zabolewicz T., 1994. Beta-lactoglobulin and kappa-casein polymorphism in relation to production traits and technological properties of milk in the herd of Polish Black-and-White cows. Genet. Pol. 35, 93-108

\section{STRESZCZENIE}

\section{Związki między genotypami i haplotypami białek mleka a wartością hodowlaną buhajów rasy polskiej czarno-biatej}

U stu czterdziestu trzech buhajów rasy polskiej czarno-białej określono genołypy w 6 punktach polimorficznych zlokalizowanych w obrębie czterech genów białek mleka: promotor genu betalaktoglobuliny (LGB-R), cxon IV genu beta-laktoglobuliny (LGB), promotor genu kappa-kazeiny (CASK-R), exon IV genu CASK (CASK), 5' flankujący region genu a!fa-laktoglobuliny (LALBA) oraz promotor genu alfa SI kazeiny (CSN1S1). Wszystkie buhaje zostały podzielone na grupy o takich samych genotypach, intragenicznych haplotypach lub kombinacjach genotypów. W każdej grupie obliczono średnią arytmetyczną i odchylenie standardowe dla następujących cech: index hodowlany (TPI), przewidywaną zdolność przekazywania (PTA) wydajności mleka, wydajności bia- 
łka, procentowej zawartości białka, wydajności tłuszczu i procentowej zawartości tłuszczu. Wartości te poddano następnie analizie wariancji w celu znalezienia istotnych związków między identyfikowanymi miejscami polimorficznymi a wartościami PTI oraz PTA. Stwierdzono statystycznie istotne różnice $(\mathrm{P}<0,01)$ pomiędzy różnymi genotypami CASK, LGB, LALBA, intragenicznymi haplotypami CASK/CASK-R, kombinacjami genotypów CASK/LALBA, CASK/LGB, LGB/LALBA, CASK/LGB/LALBA a wartościami TPI. Najwyższą wartość TPI stwierdzono u buhajów, u których zidentyfikowano następujące genotypy: CASK/LGB/LALBA AA/AA/PM, CASK/LGB AA/AA, CASK/CASK-R AA/MM, LGB/LALBA AA/MM, LGB AA, CASK/LALBA AA/MM i LALBA MM. Ponadto, wykryto związki między genotypami i kombinacjami genotypów a niektórymi wartościami PTA. 